\title{
Incremento del índice de dientes permanentes cariados, perdidos por caries y obturados, entre escolares de Loja, España
}

\author{
Diego A Imagro-N ievas, M.C., ${ }^{(1)}$ José A ntonio Benítez-Hita, M.C.,(2) María América García-A ragón, M.C., ${ }^{(2)}$ \\ María Teresa López-Lorca, Aux. Enf. ${ }^{(1)}$
}

\begin{abstract}
Almagro-Nievas D, Benítez-Hita JA, García-Aragón MA, López-Lorca MT. Incremento del índice de dientes permanentes cariados, perdidos por caries y obturados, entre escolares de Loja, España. Salud Publica Mex 2001;43:192-198. El texto completo en inglés de este artículo está
\end{abstract} disponible en: http://www.insp.mx/salud/index.html

\section{Resumen}

Objetivo. Identificar variables clínicas, socioeconómicas y de hábitos alimentarios e higiénicos, predictoras del incremento del índice (D MFT, por sus siglas en inglés) de dientes definitivos que están cariados, ausentes por caries y obturados en la cohorte de escolares de la localidad de Loja, Granada, España, en un trienio (1994-1997). Material y métodos. Estudio de cohorte retrospectivo sobre 812 alumnos a los que se les realizó una exploración dental siguiendo la metodología recomendada por la O rganización Mundial de la Salud. Para la construcción de modelos se usa la metodología de regresión logística. Resultados. El índice DMFT de toda la cohorte pasó de 1.86 a 4.5 (en el trienio y en los miembros de la cohorte de 12 años, de 3.71 a 4.47). El DMFT medio está asociado con el incremento del DMFT final. La asistencia al colegio 3 tiene un riesgo relativo (RR) de 0.69 (IC 95\% 0.60-0.8) frente a la asistencia al colegio 4 , y un RR de 0.78 (IC 95\% 0.67-0.90) frente a la asistencia al colegio 5. Conclusión. En el modelo final se encontró que el riesgo de incrementar la caries es por el DMFT medio del trienio, y que el factor protector es la asistencia al colegio 3. El texto completo en inglés de este artículo está disponible en: http://www.insp.mx/salud/ index.html

Palabras clave: caries dental; riesgo relativo; hábitos alimenticios; España

\author{
Almagro-Nievas D, Benítez-Hita JA, \\ García-Aragón MA, López-Lorca MT. \\ Increasing Decayed, Missing, \\ and Filled Teeth index among school \\ children in Loja, Spain. \\ Salud Publica Mex 2001;43:192-198. \\ The English version of this paper \\ is available at: http://www.insp.mx/salud/index.html
}

\begin{abstract}
A bstract
Objective To identify the clinical, so cioeconomic, dietary, and hygienic predictors of the increasing D ecayed, Missing, and Filled Teeth (D MFT) index, in a cohort of school children in Loja, Granada, Spain, during a three-year period (1994-1997). Material and methods A retrospective cohort study was conducted in 812 students to measure the D MFT index, using the methods recommended by theW orld H ealth 0 rganization. Statistical analysis was conducted using logistic regression models. Results. During the study period, the DMFT index increased from 1.86 to 4.5 for the study cohort as a whole, and from 3.71 to 4.47 among twelve-year olds. The D MFT at mid-period was associated to an increase in the final DMFT. The relative risk for attending school no. 3 was 0.69 ( $95 \%$ C I 0.60-0.8) vs. school no. 4 , and 0.78 ( $95 \% \mathrm{Cl} 0.67-0.90)$ vs. school no. 5. Conclusions. The final model showed that the DMFT at midperiod was a risk factor for an increase in the DMFT index and that attending school number 3 was a protective factor. The English version of this paper is available at: http:// www.insp.mx/salud/index.html
\end{abstract}

Key words: dental caries; relative risk; food habits; Spain

(1) Distrito Sanitario de Loja-Santa Fe, Granada, España.

(2) Facultad de 0 dontología, Universidad de Granada, España.

Fecha de recibido: 28 de junio de 2000 - Fecha de aprobado: 19 de diciembre de 2000 Solicitud de sobretiros: Dr. Diego Almagro N ievas. D istrito Sanitario de Loja-Santa Fe, C/ La Paz, núm. 2, Santa Fe, 18320 Granada, España. Correo electrónico:Alminero@ cica.es 
A pesar de que la salud bucodental ha experimen$A$ tado una notable mejoría en nuestro medio, ${ }^{1-3}$ la caries es la enfermedad dental más prevalente durante la infancia y continúa siendo la causa principal de pérdidas de dientes en la edad adulta.

La lucha frente a esta enfermedad depende en gran medida de factores sociales, de comportamiento y de los estilos de vida. En la población escolar española de entre 11 y 15 años, la frecuencia de cepillado dental más de una vez al día era de 35\% durante 1990, y el $78.3 \%$ ha ido alguna vez al dentista. ${ }^{4}$ La encuesta sobre hábitos de salud de los escolares andaluces, publicada en 1993, destaca que 31\% consumen diariamente dulces y pasteles, y sólo $43 \%$ de los niños de entre 6 y 8 años se cepillan los dientes a diario. ${ }^{5}$ Asimismo, en Córdoba, $80 \%$ de los alumnos de primero y cuarto grado de Enseñanza General Básica (EGB) no se cepillan a diario. ${ }^{6}$

Por ser la caries una enfermedad que se desarrolla a lo largo de los años, es importante tener en cuenta todos los posibles factores que inciden en su etiopatogenia. Un buen conocimiento de los determinantes de la salud bucodental es importante, ya que los estudios en el ámbito nacional y autonómico son más bien escasos. $^{7}$

Los criterios diagnósticos que mejor predicen hasta ahora el riesgo de caries son ${ }^{8,9}$ edad, prácticas inadecuadas de higiene oral, nivel socioeconómico bajo, historia de caries previa, índice de caries actuales, niveles de factores microbiológicos, dibujos de las caries familiares y deficiente exposición al flúor.

En la literatura es difícil encontrar modelos predictivos de caries totalmente satisfactorios. Los resultados de los diversos modelos ensayados han sido variables y poco fiables, aunque ${ }^{10,11}$ sí pueden servir para identificar grupos expuestos a un gran riesgo y que se puedan beneficiar de una actuación específica y especial. Esta enfermedad crónica, infecciosa y multifactorial es transmisible y se produce durante el periodo posteruptivo del diente. Tiene carácter dinámico, de tal forma que puede progresar más lentamente, frenarse e incluso remineralizarse o llegar a la destrucción total del diente, y sobre ella inciden ciertos factores relativos al ambiente bucal, genéticos y conductuales. ${ }^{12}$

A partir del estudio en la población escolar, se pretende valorar, en los tres años de seguimiento, la evolución de la caries y aquellos factores o variables que puedan contribuir a la identificación de los escolares que la han aumentado. Los Planes Andaluces de Salud (PAS) estimaban en 1993 y 1999 que para 2000 y 2003, el índice DMFT (número de dientes definitivos que están cariados, más los ausentes por caries y los obturados) en la población de 12 años, debía ser $\leq$ a 3 y 2 , respectivamente.

El objetivo de este trabajo es identificar variables clínicas, socioeconómicas y de hábitos alimentarios e higiénicos, predictoras del incremento del DMFT, en la cohorte de escolares de la localidad de Loja, Granada, España, en un trienio.

\section{Material y métodos}

La población de referencia es la escolar de los cinco colegios públicos de la localidad de Loja. En 1994 el total de escolares estaba entre los grados segundo y quinto de la EGB, y en 1997 la misma población escolar estaba en los cursos de quinto a octavo del mismo sistema escolar. Durante el trienio se exploró a los mismos escolares. En el apartado de resultados se describe la población que participó en todo el seguimiento ( $\mathrm{n}=816)$. La distribución por colegio, edad y sexo es bastante homogénea. Durante el trienio, el personal sanitario realizó en todos los colegios y de forma anual una intervención basada en recomendaciones sobre educación y promoción de la salud bucodental. El único criterio diferenciador en cuanto a la admisión de escolares en los centros es el lugar de la residencia en la localidad. En ningún colegio hay otras actividades específicas -colutorios, taller de técnica de cepillado, etcétera- fuera de los comunes en salud bucodental para la zona, por lo que son comparables entre ellos. Los niveles de flúor de las aguas de bebida durante 1996 fueron de $0.25 \mathrm{mg} / 1$ (ppm).

Tanto al inicio como a los tres años, se realizó una exploración oral en los escolares siguiendo la encuesta de la Organización Mundial de la Salud (OMS), ${ }^{13}$ con las modificaciones necesarias para adaptarla a lo que en este proyecto se quería investigar. Asimismo, el día del examen se llevaba a cabo una exploración visual y táctil de los niños. Previamente a este último se estandarizaron los procesos metodológicos de calibración (concordancia interobservador) con un análisis de concordancia simple.

La exploración bucodental se realizaba en los propios centros escolares. Para ello, se habilitaba el salón con más luz natural y se solicitaba a los niños que se sentaran orientados hacia las ventanas. El examen se haría de las 10:00 a las 13:00 horas, y se aplicó durante los meses de mayo y junio de 1994 y 1997. Los lunes y los viernes no se utilizaron para la exploración con el fin de evitar posibles pérdidas. Se exploró por segunda vez a 113 escolares (11.14\%); se realizó un análisis con el índice de Kappa (concordancia intraobservador), y se identificaron niveles de concordancia aceptables. ${ }^{14}$ 
La variable dependiente estudiada fue el incremento del DMFT, definida como dicotómica y categorizada como aquellos escolares que incrementaron el DMFT registrado en 1994. Como variables independientes se consideraron los colegios, la edad, el sexo, el grupo étnico (castellano y gitano), la clase social (que, con base en la profesión del jefe de familia o, en su defecto, madre o tutor/a, se le clasificó según las seis categorías que actualmente contempla la British Registrar General's Scale), ${ }^{15,16}$ hábitos higiénicos (cepillado de dientes y su frecuencia), hábitos alimentarios (consumo de golosinas y dulces fuera de las comidas), higiene bucodental (considerada por el examinador como la presencia de restos de alimentos en el reborde gingival), visitas al dentista (las veces que el niño recuerda haber ido al dentista durante el trienio del estudio), maloclusión (ausencia o presencia), índice dft (número de dientes temporales que están cariados, más los obturados), índice DMFT e índice DMFM (número de los primeros molares permanentes que están cariados, más los ausentes por caries y los obturados).

Se estudiaron dos cohortes: los que incrementaron el DMFT y los que no lo hicieron durante los tres años. Como medida de riesgo, se utilizó el riesgo relativo (RR), con un intervalo de confianza de $95 \%$ (IC 95\%). Se construyeron modelos utilizando la metodología de análisis de regresión logística, ${ }^{17}$ la cual permite valorar el efecto de las variables predictoras seleccionadas y que actúan en conjunto; como medida de asociación se utlizó la razón de momios (RM) ajustada, con un IC de 95\%. En este caso se trata de un análisis no condicional. El modelo se construyó mediante el procedimiento de Stepwise. Se consideraron los cambios de la devianza para la inclusión o exclusión del modelo, y como criterio de significación, un valor de $p<0.05$. Para el caso de variables no agrupadas, se utilizó la prueba de Wald. Los programas informáticos que se utilizaron son: Epi Info, versión 6; Epidat, versión 2.0; Glim, versión 3.77 y SPSS / PC para Windows.

El análisis de las pérdidas se realizó sobre los valores iniciales del primer examen que se aplicó en aquellos niños que no completaron el estudio y en los que sí lo hicieron (prueba de Mann-Whitney, $\mathrm{t}_{\text {exp }} \mathrm{y} \mathrm{ji}$ cuadrada).

\section{Resultados}

La distribución de los alumnos por colegio es prácticamente igual en ambos años; sólo siete de ellos cambiaron de colegio, lo que no influyó en los resultados. La distribución por sexo fue de $49.4 \%$ (403) de hombres y $50.6 \%$ (413) de mujeres. La edad media global al final del seguimiento fue de 12.14 años $(\mathrm{DE}=1.31)$. El grupo étnico gitano representó 3\% del total. La clase social IV (trabajadores parcialmente cualificados) representó $30.3 \%$, y fue la que predominó. Sólo $42.6 \%$ de los niños se cepillaba diariamente los dientes (figura 1). De la misma manera, en relación con los hábitos alimenticios, $24.6 \%$ no consumía golosinas entre comidas, y respecto a la higiene bucodental, sólo $27.6 \%$ de los niños tenían los dientes libres de restos de alimentos el día del examen (figuras 2 y 3 ). La ausencia de maloclusión en este grupo fue de $31 \%$, frente al $39.4 \%$ que estaba presente en la población total examinada. De 1994 a 1997, la media de veces que este grupo fue al dentista fue de $3.7(\mathrm{DE}=5.53)$.

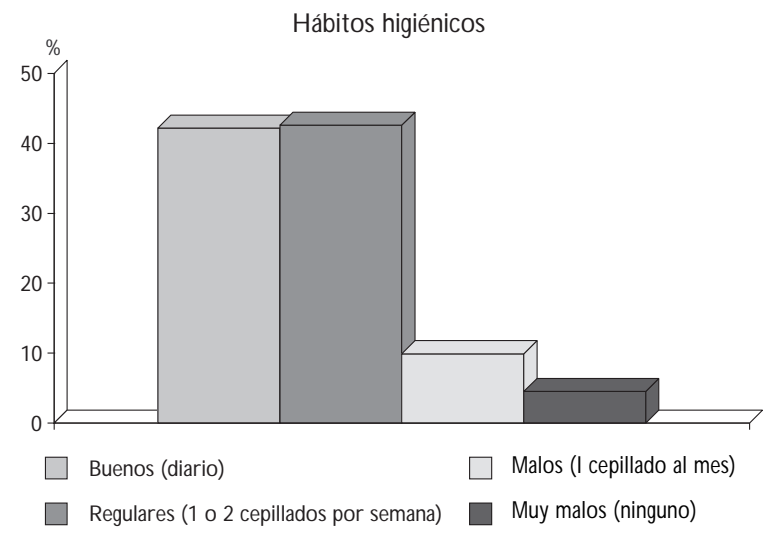

Figura 1. Distribución de la población escolar esTUDIADA, SEGÚN SUS HÁBITOS HIGIÉNICOS BUCODENTALES. LoJA, EsPAÑA, 1994 Y 1997

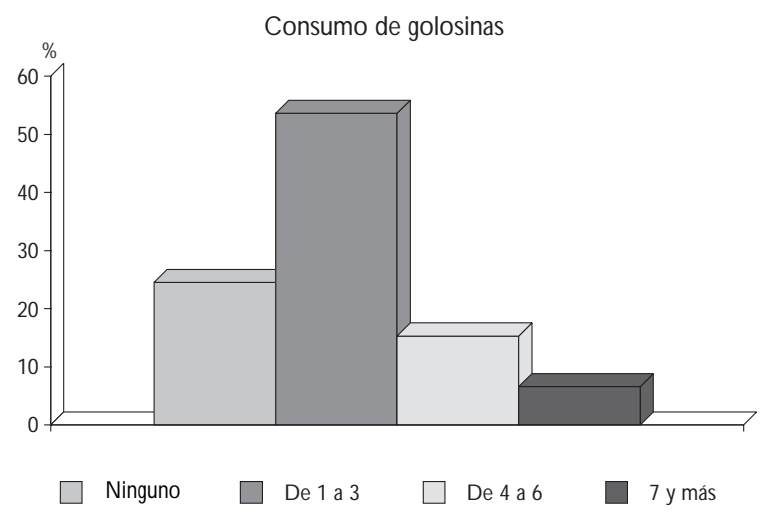

Figura 2. Distribución de la población escolar esTUDIADA SEGÚN EL CONSUMO DE GOLOSINAS ENTRE COMIDAS. LoJA, EsPAÑA, 1994 Y 1997 


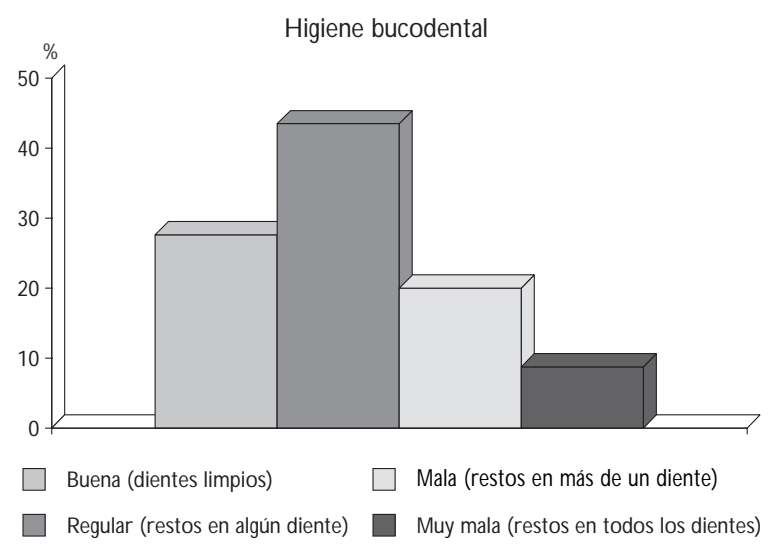

Figura 3. Distribución de la población escolar esTUDIADA SEGÚN LA HIGIENE BUCODENTAL QUE PRESENTABA. LoJA, EsPAÑA, 1994 Y 1997

El DMFT de toda la cohorte pasó de 1.86 en 1994 a 4.50 en 1997. Por grupo de edad, se observó que en los 25 escolares que habían alcanzado los 12 años en 1997, el índice se había incrementado de 3.71 a 4.47 , y en los de 10 años, de 2.11 a 2.67.

Respecto a la relación entre los que incrementaron el DMFT frente a los que no lo hicieron por colegio, se encontró un riesgo relativo (RR) de 1.27 en los colegios 1 y 2 frente al RR de 1.44 del 3 (IC 95\% 1.111.45, IC 95\% 1.23-1.68, respectivamente). La asistencia al colegio 3 tiene un RR protector frente al hecho de ser alumno del 4 y el 5 (RR 0.69, IC 95\% 0.60-0.80; RR 0.78, IC 95\% 0.67-0.90, respectivamente).

En el cuadro I se observa que, en edades más jóvenes, el RR de incrementar el DMFT es protector. No hubo diferencias significativas entre el incremento del DMFT y el sexo. El incremento del DMFT del grupo gitano frente al que no lo era, mostró un RR de 0.81 (IC 95\% 0.70-0.94). Esto quiere decir que el grupo étnico gitano tenía un riesgo mayor que el castellano de incrementar el DMFT en el trienio. No se registró una asociación significativa entre el incremento del DMFT y la clase social. Al categorizar el cepillado diario frente al no diario, no se encontró una asociación significativa con el incremento del DMFT (RR 1.10, IC 95\% 0.88-1.38). Se agrupó la categoría de no consumo de golosinas entre comidas, frente a algún otro tipo de consumo, y no se encontró asociación estadísticamente significativa con el incremento del DMFT (RR 0.80, IC 95\% 0.53-1.19). Una higiene buena frente a una regular, mala o muy mala no se asoció significativamente con el incremento del DMFT (RR 1.06, IC 95\% 0.84-1.34). La no asistencia al dentista

\section{Cuadro I \\ Riesgo relativo de incremento del DMFT, OBSERVADO ENTRE DISTINTOS GRUPOS DE EDAD de Los escolares de Loja, España, 1994-1997}

\begin{tabular}{lllll} 
Edades & No. & Porcentaje & RR & IC 95\% \\
10 & 101 & 12.4 & 8.3 & $7.0-9.8$ \\
\hline 12 & 218 & 26.7 & & \\
10 & 101 & 12.4 & 4.5 & $3.5-6.0$ \\
\hline 14 & 122 & 15.0 & & \\
11 & 170 & 20.8 & 7.7 & $6.7-8.9$ \\
\hline 14 & 122 & 15.0 & & \\
11 & 170 & 20.8 & 8.2 & $7.0-9.4$ \\
\hline 12 & 218 & 26.7 & & \\
13 & 188 & 23.01 & 8.6 & $7.6-9.7$ \\
\hline 14 & 122 & 15.0 & &
\end{tabular}

DMFT: número de dientes definitivos que están cariados, más los ausentes por caries y los obturados

RR: riesgo relativo

frente al sí, no mostró una asociación significativa con el incremento del DMFT (RR 0.86, IC 95\% 0.69-1.07). La ausencia de maloclusión frente a su presencia, no se asoció significativamente con el incremento del DMFT (RR 0.95, IC 95\% 0.75-1.20).

El total de pérdidas fue de 195 escolares, lo que representa $19.3 \%$ de la población que participó desde el inicio en el estudio en 1994. En este porcentaje se incluyó a un escolar que se negó a realizar el examen por indicación de los padres. No se observaron diferencias estadísticamente significativas entre los escolares que se exploraron dos veces y los que dejaron el estudio antes de que concluyera.

Las variables independientes incluidas en el análisis se agruparon en función de su tipo. Así, el colegio, el sexo, la clase social y el grupo étnico no van a variar desde el inicio hasta el final del estudio, pero sí los hábitos de cepillado, consumo de golosinas y dulces y la higiene bucodental, para los cuales se tomó la decisión de considerarlas al final del estudio. La edad se toma al final. Se asignó un valor promedio a los índices de caries considerados (dft, DMFT y DMFM) y a las visitas al dentista para cada uno de los expuestos. Las variables que presentaron valores mayores en los escolares con incremento del DMFT y que mostraron diferencias significativas son las que se incluyeron 
en el modelo final para los escolares explorados en dos ocasiones. Estas variables son el colegio, la edad, el grupo étnico, los hábitos higiénicos y los índices medios de caries DMFT y DMFM. El análisis multivariante se muestra en el cuadro II. La asistencia de los escolares durante los tres años al colegio 3, aparece como protectora del incremento del DMFT frente a la asistencia al colegio 1, tomado éste como referencia, de tal forma que se presentó una RM ajustada de 0.48 (IC 95\% 0.29-0.77). Como se observó, el índice medio de experiencia previa de caries y obturaciones de la dentición decidua (dft) no tiene relación estadísticamente significativa con el incremento del DMFT. La RM ajustada del DMFT medio fue de 2.2 (IC 95\% 1.752.77), lo que indica la progresión de la enfermedad. Es

\section{Cuadro II \\ RAZONes de momios aJUStAdAs POR EL INCREMENTO DEL DMFT, SEGÚN DISTINTAS VARIABLES.* LoJA, EsPAÑA, 1994 Y 1997}

\begin{tabular}{lll} 
Variable & RM ajustada & IC $95 \%$ \\
Colegio 1 & & \\
\hline Colegio 2 & 0.4199 & $0.8422-1.689$ \\
\hline Colegio 3 & 0.4816 & $0.2979-0.7792$ \\
\hline Colegio 4 & 0.999 & $0.5322-1.877$ \\
\hline Colegio 5 & 0.9564 & $0.5787-1.581$ \\
Edad & 0.9407 & $0.8204-1.079$ \\
Grupo étnico \\
Castellano
\end{tabular}

$H$. alimenticio ( $N$ o consumo de golosinas) (referencia)

\begin{tabular}{|c|c|c|}
\hline $1-3$ & 0.9353 & $0.6441-1.358$ \\
\hline $4-6$ & 1.25 & $0.6043-2.586$ \\
\hline $7-10$ & 1.679 & $0.6064-4.647$ \\
\hline $\mathrm{dft}$ & 0.9636 & $0.8541-1.087$ \\
\hline DMFT & 2.204 & $1.75-2.776$ \\
\hline DMFM & 0.7075 & $0.5513-0.908$ \\
\hline \multicolumn{3}{|c|}{$\begin{array}{l}\text { * Las variables son colegio, edad, grupo étnico, hábitos alimenticios, hi- } \\
\text { giene bucodental, visitar al dentista, índice dft (número de dientes tem- } \\
\text { porales que están cariados, más los obturados), DMFT (número de } \\
\text { dientes definitivos que están cariados, más los ausentes por caries y los } \\
\text { obturados) y D MFM (número de los primeros molares permanentes } \\
\text { que están cariados, más los ausentes por caries y los obturados) } \\
\text { Constante }\left(\beta^{\circ}\right)=0.6899 \\
\mathrm{EE}=0.8644\end{array}$} \\
\hline
\end{tabular}

decir, el riesgo de incrementar el DMFT en los escolares a los que se les realiza seguimiento es 2.2 veces mayor por cada unidad de aumento del DMFT medio de las dos exploraciones, controlando por el resto de variables independientes consideradas. No se observaron diferencias estadísticamente significativas entre el incremento del DMFT en el trienio, con la edad, el grupo étnico y los hábitos alimenticios. La variable DMFM en este caso actúa como variable confusora. La RM ajustada que presenta es de 0.70 (IC 95\% 0.55-0.90). Sin embargo, debería incrementarse al igual que lo hace el DMFT, ya que su valor está integrado en el mismo índice.

\section{Discusión}

El carácter multifactorial de las enfermedades dentales está totalmente aceptado por la comunidad científica internacional. Cualquier modelo predictivo de una enfermedad tendría que ser lo más sensible y específico para identificar de manera sencilla, eficaz y clara a los individuos que están en riesgo de enfermar, con el objeto de dirigir las medidas preventivas correspondientes en el sentido adecuado. Todo esto pone en evidencia que la relación entre las variables consideradas y la presencia o incremento de la caries es muy compleja. Es posible que se estén mezclando factores que actúan como protectores y que no estén interviniendo en el proceso de la caries y su incremento. De igual manera, no todas las causas pueden actuar de forma eficiente en cada uno de los escolares, incluso la unidad del diente, y habrá algunas que sean principales y otras secundarias o accidentales. ${ }^{18}$

Con el análisis longitudinal hecho de 1994 a 1997, se ha pretendido establecer los motivos del incremento del DMFT, en relación con las variables consideradas de exposición. Aquí se partió de escolares con una situación de DMFT que podría ser de 0 o más y que, al llegar 1997, se incrementa, por lo cual podrían existir escolares que, aun teniendo caries, no incrementen su DMFT. Este diseño permite establecer relaciones de causalidad en cuanto al aumento de caries, pero no en cuanto a la presencia de ésta.

El DMFT medio indica que el incremento de la enfermedad depende de la media del indicador DMFT entre los años de 1994 y 1997. Se está valorando la influencia de otros factores sobre la dentición permanente, y que no se controlaron en este estudio.

Se considera que los escolares que asistieron al colegio 3 durante los tres años, se protegieron del incremento del DMFT frente a los que estudiaron en el resto de los colegios. Esta diferencia entre colegios se ha encontrado entre los colegios públicos y los priva- 
dos, ${ }^{19,20}$ lo que invita a reflexionar sobre los escolares, sus familias y sus profesores. Habría que investigar, con metodología cualitativa, a los profesores como posibles agentes de salud bucodental en este colegio. Los profesionales sanitarios realizan con el mismo énfasis una serie de recomendaciones sobre hábitos en todos los colegios. El índice medio de experiencia previa de caries y obturaciones de la dentición decidua (dft) no tiene relación estadísticamente significativa con el incremento del DMFT. Es lógico pensar que en el proceso de causalidad de la caries sí intervenga la experiencia de caries pasada, pero no en cuanto al incremento de la misma, medida por el aumento del DMFT.

El número de visitas al dentista no está asociado con el incremento del DMFT, posiblemente por la forma en que se calculó la media entre el inicio del estudio y el final. El RR del incremento frente a la asistencia o no al dentista no es significativo. La variable posiblemente tenía que haber sido recogida de forma acumulativa, introduciendo en el modelo todas las visitas que ha realizado el escolar. En el incremento del DMFT tampoco interviene la edad como factor de riesgo. Posiblemente por tener poco rango como variable cuantitativa, hubiese sido necesario introducirla como variable cualitativa con distintos rangos de edad. No obstante, se observa que conforme la edad se incrementa el RR aumenta. En cuanto a la etnia gitana, hay que tener en cuenta la poca representatividad del grupo. Sin embargo, pertenecer al grupo castellano en relación con la pertenencia al grupo gitano, es protector para el incremento del DMFT (RR 0.81, IC 95\% 0.70-0.94).

La variable DMFM en este caso actúa como variable confusora. Como existe una variación muy grande entre la RM cruda y la RM ajustada (RM 1.36 y RM ajustada 0.70) hay un sesgo de confusión. El DMFM debería incrementarse al igual que lo hace el DMFT, ya que su valor está integrado en el mismo índice. La variable cumple los criterios definidos para ello ${ }^{21}$ (es un factor de riesgo de la enfermedad, ya que colabora con el incremento del DMFT; está asociada incluso con las variables de exposición que no desarrollan el efecto, y el DMFM no es un paso intermedio entre las variables de exposición y la enfermedad, sino que directamente puede causar incremento del DMFT).

El índice DMFS (superficies cariadas, ausentes y obturadas que presenta el diente permanente), que en este caso no fue estudiado, se incrementa debido a prácticas de higiene oral deficientes. ${ }^{22,23}$

En los estudios no experimentales sólo se tiene en cuenta los factores que se cree son relevantes para que se presenten caries; en los experimentales (ensa- yos comunitarios y clínicos) existe un factor de intervención por parte del investigador. Por ello es recomendable tener en cuenta, cuando se va a desarrollar una investigación, el tipo de diseño de estudio y los criterios de causalidad, ${ }^{24}$ para elegir las variables de estudio.

Hubiese sido deseable disponer de datos en las dos mediciones de los factores microbiológicos (Lactobacilos, S. Mutans) y factores salivares ( $\mathrm{pH}$, flujo, capacidad buffer, viscosidad), pero los recursos disponibles para la realización del seguimiento fueron escasos.

Como señala Rothman, ${ }^{21}$ el análisis multivariado no es una panacea estadística, y es posible encontrar una barrera para la comprensión íntima de los datos y su interpretación. Al tener en cuenta muchas variables, fue necesario realizar un análisis multivariado.

Cuando se analizó la situación de salud de los escolares, se encontraron entremezclados, en un momento dado, los efectos de múltiples determinantes y condicionantes que expresan procesos más generales del modo de vida de la localidad de Loja, procesos particulares de las condiciones de vida del grupo en cuestión y procesos singulares inherentes a estilos de vida individuales o de pequeños grupos. Es en la vida cotidiana del escolar donde se crea el espacio para intervenir de forma individual en materia de salud bucodental; asimismo, en el ámbito poblacional es necesario intervenir con campañas institucionales. ${ }^{25}$ Las variaciones y diferencias vinculadas a cambios individuales y estilos de vida se observan en el corto plazo.

$\mathrm{Al}$ igual que otros estudios, ${ }^{26,27}$ el presente trabajo se enfrentó con la dificultad de encontrar predictores de la caries en las edades estudiadas, y con la necesidad de seguir investigando con el apoyo de estos modelos.

\section{Referencias}

1.Vargas F, Robledo T, Espiga I, Hernández R. Evaluación de la salud dental en España. Madrid: Ministerio de Sanidad y Consumo, 1994.

2. Cuenca E,Alvarez MT. Evolución de la salud bucodental en España, en los últimos 20 años. Arch 0 dontoestomatol Prev Com 1991;3:45-51.

3. N oguerol B, Lodra JC, Sicilia A, Follana M. La salud bucodental en España. Antecedentes y perspectivas de futuro. Madrid: Avances MédicoDentales, 1995.

4. Ramón B, Sagrera MR, Batista JM. Conductas de los escolares españoles relacionadas con la salud (1986-1990). Madrid: Consejo Superior de Investigaciones Científicas, 1994:402.

5. Dirección General de Atención Sanitaria. Hábitos de salud de los escolares de andaluces. Sevilla: Servicio Andaluz de Salud, 1993:5.

6. Delegación Provincial de Salud y Educación y Ciencia de Córdoba. Programa de Salud Bucodental Enséñale a Sonreír. Consejería de Salud, Junta de Andalucía, 1997. 
7. Baca-G arcía P. D etección y control del paciente de riesgo. Seminario de Salud Bucodental: Prevención e innovaciones para su promoción.G ranada: EASP (D ocumento número 6), 1997.

8. Demers M, Brodeur JM, Simard PI, Mouton C, Veilleux G, Frechette S. Caries predictors suitable for mss-screenings in children:A literature review. Community Dent Health 1990;7:11-21.

9. Tinanoff N. Dental caries risk assessment and prevention. Dent Clin North Am 1995;39(4):709-719.

10. Raitio M, Pienihakkinen K, Scheinin A. Multifactorial modeling for prediction of caries increment in adolescents. Acta 0 dontol Scand 1996; 54(2):118-121.

11. World Health O rganization. Review of methods of identification of high caries risk groups and individual. Federation D entaire Internationale. Technical Report N 0. 31. Int Dent J 1988;38(3):89-177.

12. Leverett DH, Proskin HM, Keatherstone JD, A dair SM, Eisenberg AD, Mundorff-Sheretha SA et al. Caries risk assessment in a longitudinal discrimination study. J Dent Res 1993:72(2):538-543.

13. 0 rganisation Mondiale de la Santé. Enquetes sur la santé bucco-dentaire. Méthodes fondamentales. 3a. edición. Ginebra: O MS, 1988.

14. Fleiss JL. Statistical methods for rates and proportions. N ueva York: John W iley and Sons, 1984:212-225.

15. D omingo-Salvany A,A lonso JM. Propuesta de un indicador de la "clase social" basado en la ocupación. Gaceta Sanitaria 1989;10(3):420-427.

16. Grupo de Trabajo de la Sociedad Española de Epidemiología. La Medición de la clase social en ciencias de la salud. Barcelona:SG Editores, 1995: 63-66.

17. Hosmer DW, Lemeshow S. Applied logistic regressión. Nueva York: John W iley and Sons, 1989.
18. Topolsky J. Metodología de la historia. Madrid: Cátedra, 1992:198.

19. C asas VM, Gallego RM. Hábitos higiénicos bucodentales en escolares de 12 años de la ciudad de Huesca. A tencion Primaria 1992;10(6): 821-824.

20.Ayerbe MC, C aballero M, JaraA, Sanz FJ. Estudio de la caries dental en escolares de una zona urbana de la Comunidad de Madrid. Medifam 1997;7(4):226-232.

21. Rothman J. Epidemiología moderna. Díaz Santos, 1987.

22. Chestnut IG, Jones PR, Jaco bson AP, Schafer F, Stephen KW. Prevalence of clinically apparent recurrent caries in Scottish adolescents, and the influence of oral hygiene practices.University of G lasgow Dental Hospital and School, Scotland.C aries Res 1995;29(4):266-271.

23.Axelsson P, BuischiYA, Barbosa MF, Karlsson R, Prado MC. The effect of a new oral hygiene training program on approximal caries in 12-15-yearold Brazilian children: Results after three years. D epartment of Preventive Dentistry, Public D ental Health Service, Karlstad, Sweden. Adv D ent Res 1994;8(2):278-284.

24. Hill AB. The environment and disense: Asociation or caustion? Proc Royal Soc Med 1965;58:295-300.

25. Rose G. The strategies of preventive medicines. N ueva York: 0 xford University Press, 1992.

26. Raitio M, Pienihakkinen K, Scheinin A. Multifactorial modeling for prediction of caries increment in adolescents. Acta 0 dontol Scand 1996; 54(2):118-121.

27. Beck JD, D rake CW. D o root lesions tend to develop in the same people who develop coronal lesions? J Public Health Dent 1997;57(2): 82-88. 